\title{
Clinical significance of invasion distance relative to prognosis in pathological $\mathrm{T} 3$ colorectal cancer
}

\author{
MASATOSHI NOMURA $^{1 *}$, HIDEKAZU TAKAHASHI ${ }^{*}$, MAKOTO FUJII $^{2}$, NORIKATSU MIYOSHI $^{1}$, \\ NAOTSUGU HARAGUCHI ${ }^{1}$, TAISHI HATA ${ }^{1}$, CHU MATSUDA $^{1}$, HIROFUMI YAMAMOTO ${ }^{1,3}$, \\ TSUNEKAZU MIZUSHIMA ${ }^{1}$, MASAKI MORI ${ }^{4}$ and YUICHIRO DOKI ${ }^{1}$ \\ ${ }^{1}$ Department of Gastroenterological Surgery; ${ }^{2}$ Division of Health Sciences, Department of Molecular Biochemistry \\ \& Clinical Investigation, Department of Mathematical Health Science; ${ }^{3}$ Division of Health Sciences, Department \\ of Molecular Pathology, Graduate School of Medicine, Osaka University, Osaka 565-0871; ${ }^{4}$ Department of \\ Surgery and Science, Graduate School of Medical Sciences, Kyushu University, Fukuoka 812-8582, Japan
}

Received February 1, 2019; Accepted July 12, 2019

DOI: $10.3892 / \mathrm{ol} .2019 .10913$

\begin{abstract}
The T3 subdivision has been reported to predict prognosis in rectal cancer. However, few studies describe a correlation between T3 subdivision and prognosis in colon cancer. The current study aimed validate the correlation between the invasion distance (ID) beyond the muscularis propria and prognosis in colorectal cancer. The present retrospective study included 148 consecutive patients with pathologically confirmed T3 colorectal cancer, who underwent resection between January 2008 and October 2012. T3 stage was subdivided based on ID: T3a, ID $<1 \mathrm{~mm}$; T3b, $\mathrm{ID}=1-5 \mathrm{~mm}$; and T3c, ID>5 mm. Statistical analyses were performed to evaluate correlations between T3 subdivision groups (T3a $+\mathrm{T} 3 \mathrm{~b}$ versus $\mathrm{T} 3 \mathrm{c})$ and clinicopathological factors. Compared with the T3a + T3b group, the T3c group exhibited worse 3-year RFS $(\mathrm{P}=0.003)$ and 5-year CSS $(\mathrm{P}=0.006)$. Multivariate analysis demonstrated that 3 -year RFS was significantly correlated with $\operatorname{sex}(\mathrm{P}=0.03)$ and $\mathrm{ID}(\mathrm{P}=0.02)$, and 5-year CSS was significantly correlated with lymphoid dissection number $(\mathrm{P}=0.02)$ and ID $(\mathrm{P}=0.03)$. A ROC curve was constructed using ID values and recurrence data, and the area under the curve was 0.63 . These data revealed that ID beyond the muscularis propria was significantly associated with prognosis in $\mathrm{T} 3$ colorectal cancer.
\end{abstract}

Correspondence to: Dr Hidekazu Takahashi, Department of Gastroenterological Surgery, Graduate School of Medicine, Osaka University, 2-2 E-2 Yamadaoka, Suita, Osaka 565-0871, Japan

E-mail: htakahashi@gsurg.med.osaka-u.ac.jp

*Contributed equally

Key words: colorectal cancer, invasion distance, T3, prognosis, adjuvant chemotherapy

\section{Introduction}

Colorectal cancer is the third most common cancer in the United States, with an estimated 1.4 million new cases diagnosed and 0.5 million related deaths in 2017 (1). Colorectal cancer is staged based on TNM classification (2). A correlation between extramural extent and prognosis in local advanced rectal cancer was reported in 1958 (3), and the optimal cut-off points for mesorectal extension in pT3 and pT4 colorectal cancer were described in 1993 (4).

Many reports suggest that T3 subdivision might improve the prediction of prognosis in rectal cancer (5-7). A large study of colorectal cancer cases in 2007 reported a 3-year survival rate of $53 \%$ among cases with poor prognosis (T3-T4 tumors showing invasion $>5 \mathrm{~mm}$ beyond the muscularis propria) compared to $87 \%$ among cases with good prognosis (T1-2 tumors and T3 tumors showing invasion of $\leq 5 \mathrm{~mm}$ beyond the muscularis propria) (8). A recent meta-analysis concluded with an appeal to the AJCC to subdivide the T3 category with regards to rectal cancer (9).

Although the 2003 TNM Supplement, 3rd edition, suggests subdivision of the T3 classification into T3a-d (10), such subdivision is not yet specified in the 2010 7th TNM classification (UICC/AJCC). The 7th edition does include subdivision of T4 into T4a and T4b, and of Stage II into A to C. The T3 classification is also not subdivided in the 8th Japanese Classification of Colorectal Carcinoma or in the National Comprehensive Cancer Network guidelines.

While evidence suggests that T3 subdivision predicts prognosis in rectal cancer, few prior reports describe a correlation between T3 subdivision and prognosis in colon cancer. In the present study, we aimed to validate the correlation between the invasion distance (ID) beyond the muscularis propria (MP) and prognosis in colorectal cancer, excluding low rectal cancer without serosa.

\section{Materials and methods}

Study design and patient characteristics. This retrospective study included 148 consecutive patients with pathologically confirmed T3 colorectal cancer, who underwent their first operation at 
A $a-1,2$

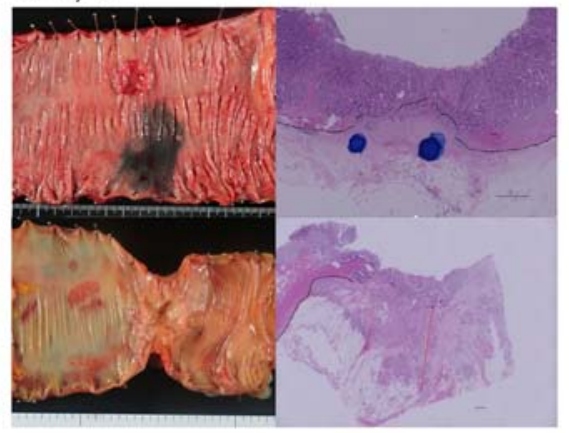

b-2

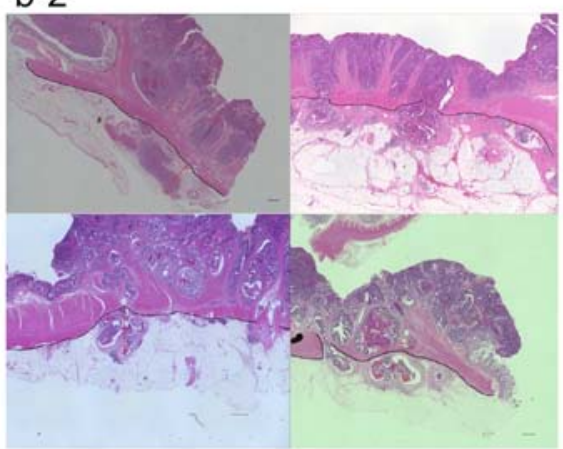

C

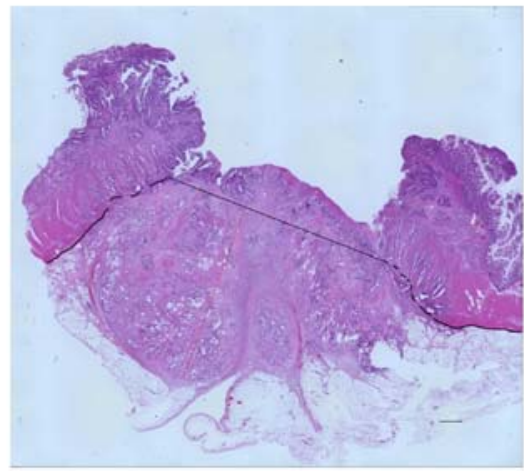

B b-1
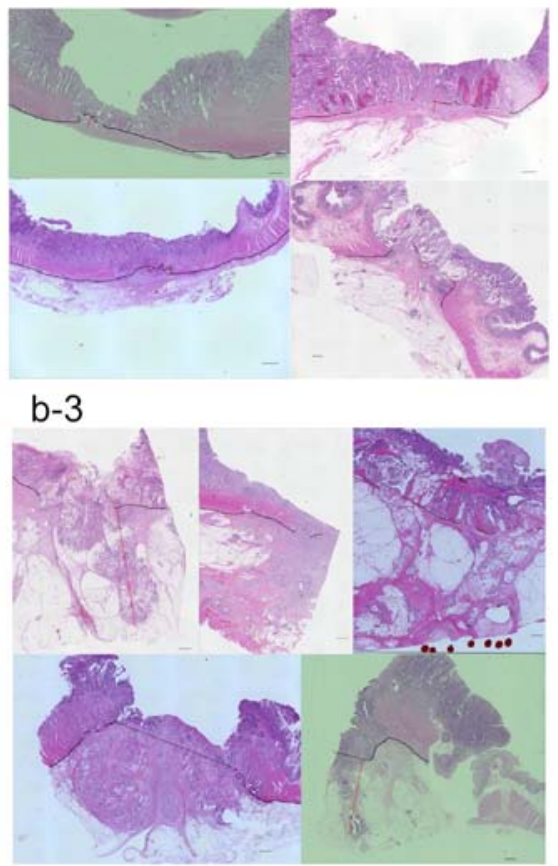

D
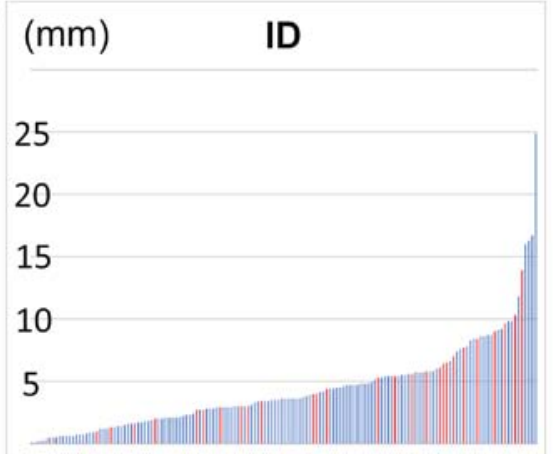

Figure 1. ID from MP line. (A) Cases of pStage II ascending colon cancer with ID values of $0.5 \mathrm{~mm}$ (a-1) and $9.8 \mathrm{~mm}$ (a-2). (B) Colorectal cancer cases classified as T3a (b-1), T3b (b-2), and T3c (b-3). Bar scale=1 mm. (C) Method for measuring maximal tumor invasion beyond the MP in cases lacking a clear MP line. The black curve underlines the muscular layer. When the muscular layer underline was unclear, an 'imagination line' (black straight line) was created by connecting the lines of the actual muscular layer. The ID was measured as the deepest distance perpendicular from the muscular layer. Scale, $1 \mathrm{~mm}$. (D) ID in all included cases. Red scale shows recurrence case. ID, invasion distance; MP, muscularis propria.

our department between January 2008 and October 2012. We excluded patients with low rectal cancer, which the deepest invasion part of the tumor occupied on the anal side of the peritoneum, and patients who received pre-operative radiotherapy and/or chemotherapy. All included patients were free of metastatic lesions, and all operations were curative.

$\mathrm{T}$ staging was determined according to the 7 th version of the UICC TNM classification of colorectal carcinoma (11). Of the 148 included patients, 118 underwent laparoscopic surgery (single-port surgery via the TANKO approach in 24 patients), and 30 underwent open surgery. Based on the degree of differentiation, tumors were divided into two groups: Well and moderately differentiated tubular adenocarcinoma, or other. All participants gave their informed consent, and this study was approved by the Institutional Review Board (approval number 15144).

Patient follow-up. After curative colorectal cancer resection, patient surveillance was based on Japanese Society for
Cancer of the Colon and Rectum (JSCCR) guidelines (12). Postoperative follow-up included serum CEA measurement every six months, CT scanning every six months, yearly colonoscopies, and routine outpatient visits. Recurrence was defined as radiologic and histopathological evidence of tumor presence after surgery. Local recurrence was defined as tumor presence at the anastomosis site, pelvis, or peritoneum.

Adjuvant chemotherapy. Adjuvant chemotherapy was recommended for all patients with stage III colorectal cancer, and was administered following JSCCR guidelines (12). For patients with high-risk colon cancer (13) and lymph node metastasis, the attending physician decided whether to administer adjuvant chemotherapy. Adjuvant chemotherapy was administered for a duration of 6 months, and the regimens included UFT, UFT + UZEL, UFT + PSK (polysaccharide-Kureha) (14), capecitabine, TS-1, FOLFOX (5-FU + oxaliplatin), and XELOX (capecitabine + oxaliplatin). 

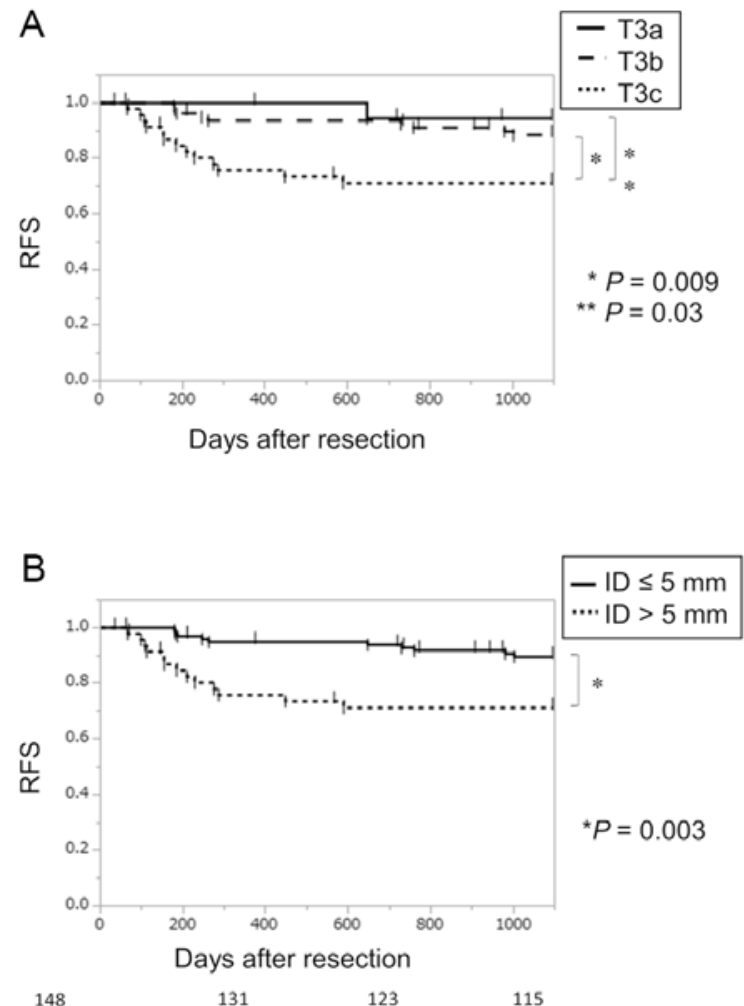
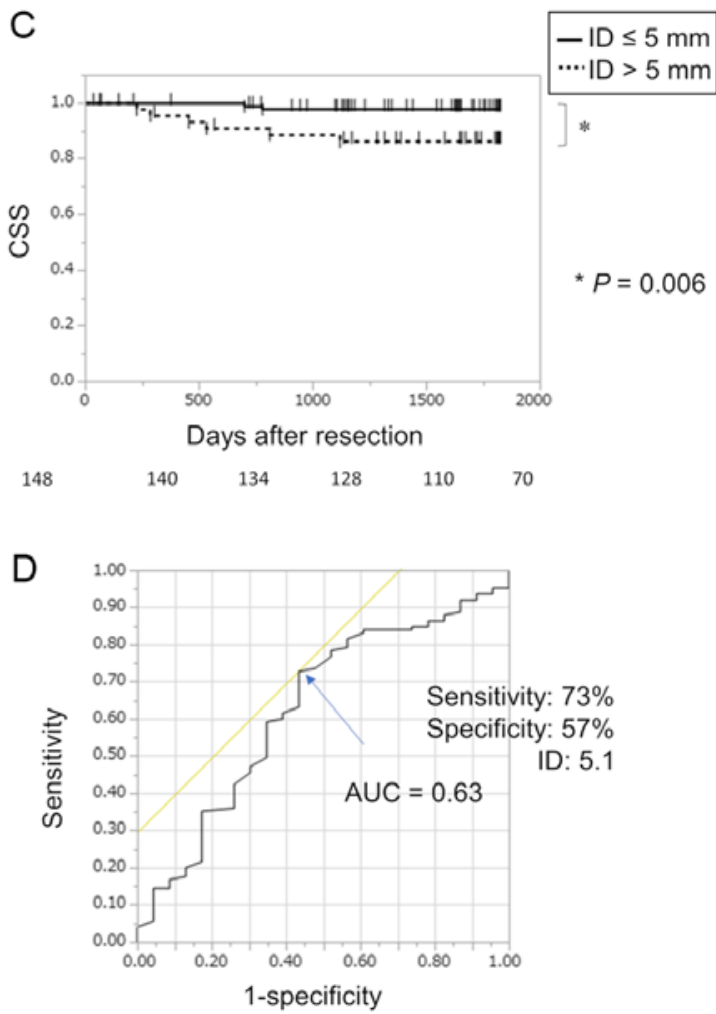

Figure 2. RFS and OS. (A) Three-year RFS compared between T3b versus T3c and between T3a versus T3c (B) and compared between cases with ID $\leq 5$ mm versus ID $>5 \mathrm{~mm}$. (C) Five-year CSS compared between cases with ID $\leq 5 \mathrm{~mm}$ versus ID $>5 \mathrm{~mm}$. receiver operating curve generated using ID to predict relapse risk. (D) Blue arrow indicates the ID cut-off. RFS, relapse-free survival; CCS, cancer-specific survival.

ID beyond the muscularis propria. Resected tumors were sliced into multiple vertical sections at the point of maximal tumor invasion, and these sections were embedded in paraffin. Next, 4- $\mu \mathrm{m}$ slices were cut and stained using hematoxylin and eosin. In at least four sections from each tumor, we measured the histological distance of the maximal tumor invasion beyond the MP, termed the ID. These measurements were taken without any clinical information about the patients (5).

Based on the ID, the pT3 stage was divided into three groups: T3a, ID $<1 \mathrm{~mm}$; T3b, ID $=1-5 \mathrm{~mm}$; and T3c, ID>5 mm. Ascending colon cancer cases categorized as T3a $(\mathrm{ID}=0.5 \mathrm{~mm})$ and $\mathrm{T} 3 \mathrm{c}(\mathrm{ID}=9.1 \mathrm{~mm})$, which were both pathological stage II are shown in Fig. 1A. The ID measurement method in the T3a, T3b, T3c groups is shown in Fig. 1B. In cases lacking a clear MP line, the maximal tumor ID beyond the MP was measured based on an imaginary line drawn horizontally extending from the normal MP lines (Fig. 1C) (5).

Statistical analysis. To assess whether the pT3 subdivision groups were correlated with clinicopathological factors, we compared the $\mathrm{T} 3 \mathrm{a}+\mathrm{T} 3 \mathrm{~b}$ group versus the $\mathrm{T} 3 \mathrm{c}$ group. We compared age, BMI, tumor size, and ID between these groups using a t test. We used Fisher's test to investigate whether the pT3 subdivision groups were correlated with sex, adjuvant chemotherapy, serum carcinoembryonic antigen (CEA) levels, degree of differentiation, lymphoid dissection number, surgical procedure, lymphoid metastasis, or angiolymphatic invasion.

Cox regression analysis was performed to analyze the independent prognostic factors for 3-year relapse-free survival (RFS) and 5-year cancer-specific survival (CSS). We investigated the correlation between pT3 group and 3-year RFS using the Wilcoxon test, and the correlation between pT3 group and 5-year CSS using the log-rank test. The ID values were used to construct receiver operating characteristic (ROC) curves, from which we determined ID cut-off values. Statistical analyses were performed using JMP Pro 13.0.0 (SAS Institute Inc., Cary, NC, USA). $\mathrm{P}<0.05$ was considered to indicate a statistically significant difference.

\section{Results}

Clinicopathological characteristics. Fig. 1D presents the ID values from all cases in this study. The pT3 subdivision was T3a in 19 patients, T3b in 81 patients, and T3c in 48 patients. The 3-year relapse rate was 5.3\% among T3a patients, $11 \%$ among T3b patients, and $27 \%$ among T3c patients. The 3 -year RFS rate was significantly worse in T3c patients compared to in T3a patients $(\mathrm{P}=0.03)$ or $\mathrm{T} 3 \mathrm{~b}$ patients $(\mathrm{P}=0.009)$ (Fig. 2A). The 3-year RFS rate was worse in the T3c group compared to in patients with an ID $\leq 5 \mathrm{~mm}$ (the $\mathrm{T} 3 \mathrm{a}+\mathrm{T} 3 \mathrm{~b}$ group) $(\mathrm{P}=0.003$; Fig. 2B).

Patients' clinicopathological characteristics are shown in Table I. Table II shows the correlations between 3-year RFS and clinicopathological characteristics, as determined by univariate and multivariate analysis. Univariate analysis revealed that 3 -year RFS was correlated with sex $(\mathrm{P}=0.03)$, vascular invasion $(\mathrm{P}=0.02)$, lymphoid metastasis $(\mathrm{P}=0.02)$, and ID with a cut-off point of $5 \mathrm{~mm}(\mathrm{P}=0.005)$. Multivariate analysis showed that 3-year RFS was independently correlated with sex $(P=0.03)$ and ID $(P=0.02)$. 
Table I. Clinicopathological characteristics of the T3c and T3ab groups.

\begin{tabular}{|c|c|c|c|c|}
\hline Characteristics & All patients $(n=148)$ & $\mathrm{T} 3 \mathrm{c}(>5 \mathrm{~mm})(n=48)$ & $\mathrm{T} 3 \mathrm{ab}(\leq 5 \mathrm{~mm})(n=100)$ & P-value \\
\hline Age (years) & $66.0 \pm 11.3$ & $66.5 \pm 11.1$ & $65.7 \pm 11.4$ & 0.68 \\
\hline \multicolumn{5}{|l|}{ Sex } \\
\hline Male & 87 & 29 & 58 & \multirow[t]{2}{*}{0.78} \\
\hline Female & 61 & 19 & 42 & \\
\hline BMI & $22.5 \pm 4.2$ & $22.3 \pm 4.9$ & $22.6 \pm 3.8$ & 0.77 \\
\hline \multicolumn{5}{|l|}{ Tumor location } \\
\hline $\mathrm{C}$ & 12 & 8 & 4 & - \\
\hline A & 31 & 9 & 22 & - \\
\hline $\mathrm{T}$ & 15 & 8 & 7 & - \\
\hline $\mathrm{D}$ & 4 & 1 & 3 & - \\
\hline $\mathrm{S}$ & 44 & 9 & 35 & - \\
\hline $\mathrm{RS}$ & 14 & 3 & 11 & - \\
\hline $\mathrm{Ra}$ & 28 & 10 & 18 & - \\
\hline Right & 58 & 25 & 33 & 0.03 \\
\hline Left & 90 & 23 & 67 & \\
\hline \multicolumn{5}{|c|}{ Adjuvant chemotherapy } \\
\hline Yes & 53 & 22 & 31 & \multirow[t]{2}{*}{0.08} \\
\hline No & 95 & 26 & 69 & \\
\hline \multicolumn{5}{|c|}{ Serum CEA levels (ng/ml) } \\
\hline$>5$ & 62 & 19 & 43 & \multirow[t]{2}{*}{0.66} \\
\hline$\leq 5$ & 85 & 29 & 56 & \\
\hline \multicolumn{5}{|c|}{ Degree of differentiation } \\
\hline por, sig, muc & 16 & 7 & 9 & \multirow[t]{2}{*}{0.33} \\
\hline tub & 131 & 41 & 90 & \\
\hline Size $(\mathrm{mm})$ & $48.2 \pm 21.0$ & $59.3 \pm 26.0$ & $42.9 \pm 15.6$ & 0.0002 \\
\hline \multicolumn{5}{|c|}{ Lymphnoid dissection number } \\
\hline$\geq 12$ & 125 & 41 & 84 & \multirow[t]{2}{*}{0.82} \\
\hline$<12$ & 23 & 7 & 16 & \\
\hline \multicolumn{5}{|c|}{ Surgical procedure } \\
\hline Laparoscopy & 118 & 38 & 80 & \multirow[t]{2}{*}{0.91} \\
\hline Open & 30 & 10 & 20 & \\
\hline \multicolumn{5}{|c|}{ Lymphnoid metastasis } \\
\hline $\mathrm{N}(+)$ & 64 & 28 & 36 & \multirow[t]{2}{*}{0.01} \\
\hline $\mathrm{N}(-)$ & 84 & 20 & 64 & \\
\hline \multicolumn{5}{|c|}{ Angiolymphatic invasion } \\
\hline $\operatorname{ly}(+)$ & 130 & 46 & 84 & \multirow[t]{2}{*}{0.03} \\
\hline ly(-) & 18 & 2 & 16 & \\
\hline $\mathrm{v}(+)$ & 59 & 25 & 34 & \multirow[t]{2}{*}{0.04} \\
\hline $\mathrm{v}(-)$ & 89 & 23 & 66 & \\
\hline ID (mm) & $4.4 \pm 3.6$ & $8.2 \pm 3.8$ & $2.5 \pm 1.4$ & $<0.0001$ \\
\hline
\end{tabular}

Data are presented as mean \pm SD or number of patients. C, Cecum; A, Ascending colon; T, Transverse colon; D, Descending colon; S, Sigmoid colon; RS, Rectosigmoid colon; Ra, Rectum above; CEA, carcinoembryonic antigen; tub, tubular adenocarcinoma; por, poorly differentiated adenocarcinoma; sig, signet ring cell carcinoma; muc, mucinous adenocarcinoma; ID, invasion distance beyond MP; N+ lymph node metstasis presence; $\mathrm{N}$ - lymph node metastasis absence; ly+ lymphatic invasion presence; ly- lymphatic invasion absence; v+ venous invasion presence; $\mathrm{v}$ - venous invasion absence.

The 5-year CSS rate was worse among patients with ID $>5 \mathrm{~mm}$ compared to those with $\mathrm{ID} \leq 5 \mathrm{~mm}(\mathrm{P}=0.006)$ (Fig. 2C). Univariate analysis revealed that 5-year CSS was correlated with lymphoid dissection number $(\mathrm{P}=0.02)$, vascular invasion $(\mathrm{P}=0.03)$, and ID $(\mathrm{P}=0.009$; Table III). In multivariate analysis, 5-year CSS was significantly 
Table II. Risk factors for postoperative recurrence in all patients using univariate and multivariate analysis.

\begin{tabular}{|c|c|c|c|c|c|c|}
\hline \multirow[b]{2}{*}{ Characteristics } & \multicolumn{3}{|c|}{ Univariate } & \multicolumn{3}{|c|}{ Multivariate } \\
\hline & Hazard ratio & $95 \% \mathrm{CI}$ & P-value & Hazard ratio & $95 \% \mathrm{CI}$ & P-value \\
\hline \multicolumn{7}{|l|}{ Sex } \\
\hline Male/female & 2.80 & $1.12-8.50$ & 0.03 & 2.76 & $1.09-8.41$ & 0.03 \\
\hline \multicolumn{7}{|l|}{ Location } \\
\hline Left/right & 1.24 & $0.54-3.09$ & 0.62 & & & \\
\hline \multicolumn{7}{|c|}{ Serum CEA levels (ng/ml) } \\
\hline$>5 / \leq 5$ & 1.80 & $0.79-4.22$ & 0.16 & & & \\
\hline \multicolumn{7}{|l|}{ Surgical procedure } \\
\hline Laparoscopy/open & 0.87 & $0.35-2.62$ & 0.78 & & & \\
\hline \multicolumn{7}{|c|}{ Lymphoid dissection number } \\
\hline$\geq 12 /<12$ & 0.52 & $0.22-1.44$ & 0.19 & & & \\
\hline \multicolumn{7}{|c|}{ Angiolymphatic invasion } \\
\hline ly(+)/ly(-) & 3.42 & $0.72-61.3$ & 0.14 & & & \\
\hline $\mathrm{v}(+) / \mathrm{v}(-)$ & 2.60 & $1.14-6.24$ & 0.02 & 1.91 & $0.82-4.66$ & 0.14 \\
\hline \multicolumn{7}{|l|}{ Lymphoid metastasis } \\
\hline $\mathrm{N}(+) / \mathrm{N}(-)$ & 2.63 & $1.14-6.53$ & 0.02 & 1.99 & $0.84-5.05$ & 0.12 \\
\hline \multicolumn{7}{|c|}{ Degree of differentiation } \\
\hline por, sig, muc/tub & 1.87 & $0.54-4.97$ & 0.29 & & & \\
\hline \multicolumn{7}{|c|}{ Adjuvant chemotherapy } \\
\hline Yes/no & 0.53 & $0.23-1.21$ & 0.13 & & & \\
\hline \multicolumn{7}{|l|}{ ID (mm) } \\
\hline$>5 \mathrm{~mm} / \leq 5 \mathrm{~mm}$ & 3.32 & $1.46-7.79$ & 0.005 & 2.82 & $1.22-6.73$ & 0.02 \\
\hline
\end{tabular}

tub, tubular adenocarcinoma; por, poorly differentiated adenocarcinoma; sig, signet ring cell carcinoma; muc, mucinous adenocarcinoma; ID, invasion distance beyond MP; CI, confidence interval; N+ lymph node metstasis presence; $\mathrm{N}$ - lymph node metastasis absence; ly+ lymphatic invasion presence; ly- lymphatic invasion absence; v+ venous invasion presence; v- venous invasion absence.

correlated with lymphoid dissection number $(\mathrm{P}=0.02)$ and ID $(\mathrm{P}=0.03)$.

Recurrence and cancer-specific survival. Recurrence occurred in 27 patients, with 28 sites affected during the first recurrences after surgery. Of these 27 patients, 21 exhibited distant metastases (14 liver, 8 lung) and 6 exhibited local recurrence (3 lymph node, 2 peritoneal, 1 anastomotic recurrence). Of the 148 included patients, 23 died, including 9 deaths due to colorectal cancer.

Adjuvant chemotherapy. Adjuvant chemotherapy was administered to 53 patients. Decisions regarding adjuvant chemotherapy were made by the physicians, with consideration of the patients' wishes. The adjuvant therapy regimen was UFT + UZEL in 28 patients, UFT + PSK in 6 patients, capecitabine in 5 patients, XELOX in 5 patients, and other regimens in 9 patients.

ID cut-off value. An ROC curve was constructed using ID values and recurrence data, and the area under the curve was 0.63 (Fig. 2D). The optimal cut-off value for separating recurrence from non-recurrence was an ID of $5.1 \mathrm{~mm}$ (73\% sensitivity, $57 \%$ specificity, and $70 \%$ accuracy).

\section{Discussion}

Studies of colorectal cancer report a predominance of the T3 stage, with the following T-stage distribution: $12 \% \mathrm{~T} 1,14 \%$ $\mathrm{T} 2,44 \% \mathrm{~T} 3,24 \% \mathrm{~T} 4 \mathrm{a}$, and $6 \% \mathrm{~T} 4 \mathrm{~b}$ (12). Similarly, in our department, the vast majority of stage II and III colorectal cancer cases are categorized as T3. The 4th TNM classification supplementary for low rectal cancer mentions T3 subdivision as an optional classification. However, the 7 th TNM classification from 2010 recommends subdivision of T4 cases into T4a and T4b cases, while T3 cases still constitute a single category. Many reports describe rectal cancer cases with a great mesorectal extension depth has having a poor prognosis, and clinical trials of neoadjuvant chemotherapy in colon cancer have used an ID of $\geq 5 \mathrm{~mm}$ to delineate the poor prognostic group (15). However, T3 subdivision in colon cancer is still not common, and few reports have actually validated the prognostic influence using pathological analysis.

In our present study, we evaluated the clinical significance of T3 subdivision based on ID in colorectal cancer, excluding low rectal cancer. We found that an ID of $5.1 \mathrm{~mm}$ was the optimal cut-off value for separating recurrence from non-recurrence (Fig. 2D), and thus used an ID of $5 \mathrm{~mm}$ as the cut-off value 
Table III. Risk factors for CSS in all patients using univariate and multivariate analysis

\begin{tabular}{|c|c|c|c|c|c|c|}
\hline \multirow[b]{2}{*}{ Characterisitics } & \multicolumn{3}{|c|}{ Univariate } & \multicolumn{3}{|c|}{ Multivariate } \\
\hline & Hazard ratio & $95 \%$ CI & P-value & Hazard ratio & $95 \% \mathrm{CI}$ & P-value \\
\hline \multicolumn{7}{|l|}{ Sex } \\
\hline Male/female & 5.05 & $0.89-94.4$ & 0.07 & & & \\
\hline $\begin{array}{l}\text { Location } \\
\text { Left/right }\end{array}$ & 1.09 & $0.27-5.31$ & 0.91 & & & \\
\hline \multicolumn{7}{|c|}{ Serum CEA levels (ng/ml) } \\
\hline$>5 / \leq 5$ & 0.84 & $0.17-3.43$ & 0.81 & & & \\
\hline \multicolumn{7}{|l|}{ Surgical procedure } \\
\hline \multicolumn{7}{|c|}{ Lymphoid dissection number } \\
\hline \multicolumn{6}{|c|}{ Angiolymphatic invasion } & \\
\hline $\mathrm{v}(+) / \mathrm{v}(-)$ & 4.86 & $1.12-33.2$ & 0.03 & 3.19 & $0.71-22.2$ & 0.13 \\
\hline \multicolumn{7}{|l|}{ Lymphoid metastasis } \\
\hline $\mathrm{N}(+) / \mathrm{N}(-)$ & 4.08 & $0.94-27.8$ & 0.06 & & & \\
\hline \multicolumn{6}{|c|}{ Degree of differentiation } & \\
\hline $\begin{array}{l}\text { Adjuvant chemother } \\
\text { Yes/no }\end{array}$ & 0.57 & $0.08-2.48$ & 0.47 & & & \\
\hline \multicolumn{7}{|l|}{$\mathrm{ID}(\mathrm{mm})$} \\
\hline$>5 \mathrm{~mm} / \leq 5 \mathrm{~mm}$ & 4.19 & $1.03-20.4$ & 0.009 & 6.19 & $1.39-43.1$ & 0.03 \\
\hline
\end{tabular}

CSS, cancer-specific survival; tub, tubular adenocarcinoma; por, poorly differentiated adenocarcinoma; sig, signet ring cell carcinoma; muc, mucinous adenocarcinoma; ID, invasion distance beyond MP; CI, confidence interval; N+ lymph node metstasis presence; $\mathrm{N}$ - lymph node metastasis absence; ly+ lymphatic invasion presence; ly-lymphatic invasion absence; $v+$ venous invasion presence; $v$ - venous invasion absence.

for analysis. This was similar to previous studies, which have performed T3 subdivision as follows: T3a, ID $<1 \mathrm{~mm}$; T3b, ID=1-5 mm; T3c, 5<ID $\geq 15 \mathrm{~mm}$; and T3d, >15 mm. In an analysis of rectal cancer, Shin et al compared prognosis between the $\mathrm{T} 3 \mathrm{a}+\mathrm{T} 3 \mathrm{~b}$ group and the $\mathrm{T} 3 \mathrm{c}+\mathrm{T} 3 \mathrm{~d}$ group (6). Zinicola et al retrospectively summarized 12 studies reporting an ID cut-off in T3 rectal cancer, and noted that the smallest standard errors were found when using a 4-mm or 5-mm ID cut-off to analyze 5-year survival (9). In one of the included studies, Merkel et al used a 5-mm ID cut-off in 853 patients from the Study Group for Colo-Rectal Carcinoma (16). Finally, in an analysis of preoperative diagnosis using CT or MRI, a 5-mm cut-off was used as a criterion for surgery or neoadjuvant chemotherapy in colon cancer (15).

Standard therapy for Stage III colon cancer includes colon resection with mesentery (17) and oxaliplatin-based adjuvant chemotherapy (18-20). In cases with lymph node metastasis, JSCCR guidelines recommend adjuvant chemotherapy (12). Our present results indicated that T3 subdivision (T3c versus $\mathrm{T} 3 \mathrm{a}+\mathrm{T} 3 \mathrm{~b})$ correlated with tumor size $(\mathrm{P}=0.0002)$, lymphoid metastasis $(\mathrm{P}=0.01)$, lymphatic invasion $(\mathrm{P}=0.03)$, and vascular invasion $(\mathrm{P}=0.04)$. Adjuvant chemotherapy was more commonly administered in the $\mathrm{T} 3 \mathrm{c}$ group than in the $\mathrm{T} 3 \mathrm{a}+\mathrm{T} 3 \mathrm{~b}$ group $(\mathrm{P}=0.08)$. In other words, $\mathrm{T} 3$ subdivision was significantly correlated with prognostic factors, and was associated with the administration of adjuvant chemotherapy. We found that ID and sex were independent risk factors for 3-year RFS, and that ID and lymphoid dissection number were independent risk factors for 5-year CSS (Tables II, III). Overall, T3c cases had a worse prognosis than T3a and T3b cases (Fig. 2A). Lymphoid metastasis, degree of differentiation, and angiolymphatic invasion were not independent risk factors for 3-year RFS or 5-year CSS in our study, likely due to the inclusion of patients with and without adjuvant chemotherapy. It might be useful to use ID as a prognostic marker for T3 colorectal cancer patients, except those with low rectal cancer, without accounting for adjuvant chemotherapy.

Among the 64 patients in our study with lymph node metastasis, 18 (28\%) were not administered adjuvant chemotherapy. Of these 18 patients, 6 suffered a relapse, including $4 \mathrm{~T} 3 \mathrm{c}$ and $2 \mathrm{~T} 3 \mathrm{~b}$ cases. These data suggest that adjuvant chemotherapy should be administered in T3c cases with lymph node metastasis. Among the 84 patients without lymph node metastasis, 7 (8\%) were administered adjuvant chemotherapy. No deaths due to colorectal cancer occurred among the T3a cases in our study. We found that 3-year RFS was significantly worse among T3c cases than T3b cases $(\mathrm{P}=0.009)$ (Fig. 2A). Since operative stress carries a risk of promoting tumor growth, immediate intervention is required 
to eradicate micrometastases (21-25). Our present data suggest that it might be beneficial to administer earlier treatment, such as neoadjuvant chemotherapy, in T3c cases.

Our present study had three limitations. First, it was a retrospective study with a small number of cases. Second, the adjuvant chemotherapy methods were diverse, and the administration of adjuvant chemotherapy was decided by the attending physician. Third, there was only a small number of events for the 5 -year CSS analysis (9 patients). The reason for the difference in gender is unclear. We have to increase the number of the cases and to consider again in the future, and to plan prospective study about the problem of postoperative adjuvant chemotherapy.

Among cases of T3 colorectal cancer (excluding low rectal cancer), ID above the muscularis propria was significantly associated with prognosis.

\section{Acknowledgements}

Not applicable

\section{Funding}

No funding was received.

\section{Availability of data and materials}

All data generated or analyzed during the present study are included in this published article.

\section{Authors' contributions}

MN, HT, and MF conceived and designed the study, and acquired the data. MN, HT, MF, NM, NH, TH, CM, HY, TM, $\mathrm{MM}$ and YD analyzed and interpreted the data. MN and HT drafted the article. All authors critically revised the article for important intellectual content, and approved the final version of the article to be published.

\section{Ethics approval and consent to participate}

Informed consent was obtained from all individual participants included in the current study. The present study was approved by the Institutional Review Board (approval no. 15144).

\section{Patient consent for publication}

Not applicable.

\section{Competing interests}

The authors declare that they have no competing interests.

\section{Reference}

1. Siegel RL, Miller KD and Jemal A: Cancer statistics, 2017. CA Cancer J Clin 67: 7-30, 2017.

2. Edge SB, Byrd DR, Compton CC, Fritz AG, Greene FL and Trotti A (eds): AJCC Cancer Staging Mannual. 7th edition. Springer, New York, NY, 2010.

3. Dukes CE and Bussey HJ: The spread of rectal cancer and its effect on prognosis. Br J Surg 12: 309-320, 1958.

4. Hermanek P: TNM Supplement 1993: A commentary on uniform Use. Berlin: Springer-Verlag, 1993.
5. Shirouzu K, Akagi Y, Fujita S, Ueno H, Takii Y, Komori K, Ito $M$ and Sugihara K; Japanese Society for Cancer of the Colon and Rectum (JSCCR) on clinical significance of the mesorectal extension of rectal cancer: Clinical significance of the mesorectal extension of rectal cancer: A japanese multi-institutional study. Ann Surg 253: 704-710, 2011.

6. Shin R, Jeong SY, Yoo HY, Park KJ, Heo SC, Kang GH, Kim WH and Park JG: Depth of mesorectal extension has prognostic significance in patients with T3 rectal cancer. Dis Colon Rectum 55: 1220-1228, 2012.

7. Sueda T, Ohue M, Noura S, Shingai T, Nakanishi K and Yano M: Prognostic significance of a preoperative magnetic resonance imaging assessment of the distance of mesorectal extension in clinical T3 lower rectal cancer. Surg Today 46: 1249-1257, 2016.

8. Smith NJ, Bees N, Barbachano Y, Norma AR, Swift RI and Brown G: Preoperative computed tomography staging of nonmetastatic colon cancer predicts outcome: Implications for clinical trials. Br J Cancer 96: 1030-1036, 2007.

9. Zinicola R, Pedrazzi G, Haboubi N and Nicholls RJ: The degree of extramural spread of $\mathrm{T} 3$ rectal cancer: An appeal to the American joint committee on cancer. Colorectal Dis 19: 8-15, 2017.

10. Wittekind C: TNM supplement: A commentary on uniform use. 3rd edition. Hoboken: Wiley-Liss, 2003.

11. Sobin LH, Gospodarowicz MK and Wittekind C (eds): UICC TNM Classification of Malignant Tumors. 7th edition. WileyBlackwell, New York, NY, 2009.

12. Watanabe T, Itabashi M, Shimada Y, Tanaka S, Ito Y, Ajioka Y, Hamaguchi T, Hyodo I, Igarashi M, Ishida H, et al: Japanese society for cancer of the colon and rectum (JSCCR) guidelines 2014 for the treatment of colorectal cancer. Int J Clin Oncol 20: 207-239, 2015.

13. Kumar A, Knnecke HF, Renouf DJ, Lim HJ, Gill S, Woods R, Speers C and Cheung WY: Adjuvant chemotherapy use and outcomes of patients with high-risk versus low-risk stage II colon cancer. Cancer 121: 527-534, 2015.

14. Yoshitani S and Takashima S: Efficacy of postoperative UFT (Tegafur/Uracil) plus PSK therapies in elderly patients with resected colorectal cancer. Cancer Biother Radiopharm 24: 35-40, 2009.

15. Foxtrot Collaborative Group: Feasibility of preoperative chemotherapy for locally advanced, operable colon cancer: The pilot phase of a randomised controlled trial. Lancet Oncol 13: 1152-1160, 2012.

16. Merkel S, Mansmann U, Siassi M, Papadopoulos T, Hohenberger $\mathrm{W}$ and Hermanek P: The prognostic inhomogeneity in pT3 rectal carcinomas. Int J Colorectal Dis 16: 298-304, 2001.

17. Bertelsen CA, Neuenschwander AU, Jansen JE, Wihelmsen M, Kirkegaard-Klitbo A, Tenma JR, Bols B, Ingeholm P, Rasmussen LA, Jepsen LV, et al: Disease-free survival after complete mesocolic excision compared with conventional colon cancer surgery: A retrospective, population-based study. Lancet Oncol 16: 161-168, 2015.

18. Taieb J, Tabernero J, Mini E, SubitF, Folprecht G, Van Laethem JL, Thaler J, Bridgewater J, Petersen LN, Blons H, et al: Oxaliplatin, fluorouracil, and leucovorin with or without cetuximab in patients with resected stage III colon cancer (PETACC-8): An open-label, randomized phase 3 trial. Lancet Oncol 15: 862-873, 2014.

19. Colvin H, Mizushima T, Eguchi H, Takiguchi S, Doki Y and Mori M: Gastroenterological surgery in Japan: The past, the present and the future. Ann Gastroenterol Surg 1: 5-10, 2017.

20. Matsuda T, Yamashita K, Hasegawa H, Oshikiri T, Hosono M, Higashino N, Yamamoto M, Matsuda Y, Kanaji S, Nakamura T, et al: Recent updates in the surgical treatment of colorectal cancer. Ann Gastroenterol Surg 2: 129-136, 2018.

21. Finlay IG, Meek D, Brunton F and McArdle CS: Growth rate of hepatic metastases in colorectal carcinoma. Br J Surg 75: 641-644, 1988.

22. Tanaka K, Shimada H, Miura M, Fujii Y, Yamaguchi S, Endo I, Sekido H, Togo S and Ike H: Metastatic tumor doubling time: Most important prehepatectomy predictor of survival and non-recurrence of hepatic colorectal cancer metastasis. World J Surg 28: 263-270, 2004.

23. Zeamari S, Roos E and Stewart FA: Tumour seeding in peritoneal wound sites in relation to growth-factor expression in early granulation tissue. Eur J Cancer 40: 1431-1440, 2004.

24. Fahmy RG, Dass CR, Sun LQ, Chesterman CN and Khachigian LM: Transcription factor Egr-1 supports FGF-dependent angiogenesis during neovascularization and tumor growth. Nat Med 9: 1026-1032, 2003.

25. Schelfhout VR, Coene ED, Delaey B, Waeytens AA, De Rycke L, Deleu M and De Potter CR: The role of heregulin-alpha as a motility factor and amphiregulin as a growth factor in wound healing. J Pathol 198: 523-533, 2002. 